\title{
Observation of Polar Vortices in Oxide Superlattices
}

R. Ramesh ${ }^{1,2,3}$

${ }^{1}$ Department of Materials Science and Engineering, University of California, Berkeley, CA, USA

${ }^{2}$ Department of Physics, University of California, Berkeley, CA, USA

${ }^{3}$ Materials Sciences Division, Lawrence Berkeley National Laboratory, Berkeley, CA, USA

The complex interplay of spin, charge, orbital, and lattice degrees of freedom has provided for a plethora of exotic phase and physical phenomena ${ }^{1,2,3,4,5}$. Among these, in recent years, topological states of matter and spin textures have emerged as fascinating consequences of the electronic band structure and the interplay between spin and spin-orbit coupling in materials ${ }^{6,7}$. In this work, we leverage the competition between charge, orbital, and lattice degrees of freedom in superlattices of $\mathrm{PbTiO}_{3} / \mathrm{SrTiO}_{3}$ to produce complex, vortex-antivortex pairs (that exhibit smoothly varying ferroelectric polarization with a $10 \mathrm{~nm}$ periodicity) that are reminiscent of topological features such as skyrmions and merons ${ }^{6}$. Using a combination of advanced layer-by-layer growth techniques, atomic-resolution mapping of structure and local polar distortions using scanning-transmission electron microscopy, and phase-field modeling approaches we present a comprehensive picture of the nature of the varying polarization profile in such vortex states. The continuous rotation of the polar state into the vortex structures is thought to occur from an interplay of polar discontinuities at the $\mathrm{PbTiO}_{3} / \mathrm{SrTiO}_{3}$ interface (where $\nabla \cdot P \neq 0$ ), the phase transformation strain and gradient energy in the $\mathrm{PbTiO}_{3}$ layer, and the strain imposed by the substrate. Finally, the implications of these observations are discussed as they pertain to producing new states of matter and phenomena in ferroic materials. 
https://doi.org/10.1017/S1431927616007078 Published online by Cambridge University Press 